\title{
Exponential Distribution
}

National Cancer Institute

\section{Source}

National Cancer Institute. Exponential Distribution. NCI Thesaurus. Code C53222.

A family of continuous probability distribution functions, whose shape is determined by its rate parameter. It has been described as the continuous analog of the geometric distribution. 